\title{
Durable Clinical Benefit With Nivolumab Plus Ipilimumab in DNA Mismatch Repair-Deficient/Microsatellite Instability-High Metastatic Colorectal Cancer
}

Michael J. Overman, Sara Lonardi, Ka Yeung Mark Wong, Heinz-Josef Lenz, Fabio Gelsomino, Massimo Aglietta, Michael A. Morse, Eric Van Cutsem, Ray McDermott, Andrew Hill, Michael B. Sawyer, Alain Hendlisz, Bart Neyns, Magali Svrcek, Rebecca A. Moss, Jean-Marie Ledeine, Z. Alexander Cao, Shital Kamble, Scott Kopetz, and Thierry André

Author affiliations and support information (if applicable) appear at the end of this article.

Published at jco.org on January 20, 2018. Clinical trial information: NCT02060188.

Corresponding author: Michael J. Overman, MD, University of Texas MD Anderson Cancer Center, 1515 Holcombe Blvd, Houston, TX 77030; e-mail: moverman@mdanderson.org.

(C) 2018 by American Society of Clinical Oncology

0732-183X/18/3608w-773w/\$20.00

\section{ASSOCIATED CONTENT}

Q Appendix DOI: https://doi.org/10.1200/JCO 2017.76.9901

DOI: https://doi.org/10.1200/JCO.2017 76.9901

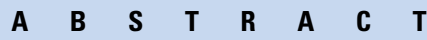

\section{Purpose}

Nivolumab provides clinical benefit (objective response rate [ORR], 31\%; 95\% Cl, 20.8 to 42.9; disease control rate, $69 \%$; 12-month overall survival [OS], 73\%) in previously treated patients with DNA mismatch repair-deficient (dMMR)/microsatellite instability-high (MSI-H) metastatic colorectal cancer (mCRC); nivolumab plus ipilimumab may improve these outcomes. Efficacy and safety results for the nivolumab plus ipilimumab cohort of CheckMate-142, the largest single-study report of an immunotherapy combination in $\mathrm{dMMR/MSI-H} \mathrm{mCRC}$, are reported.

\section{Patients and Methods}

Patients received nivolumab $3 \mathrm{mg} / \mathrm{kg}$ plus ipilimumab $1 \mathrm{mg} / \mathrm{kg}$ once every 3 weeks (four doses) followed by nivolumab $3 \mathrm{mg} / \mathrm{kg}$ once every 2 weeks. Primary end point was investigator-assessed ORR.

\section{Results}

Of 119 patients, $76 \%$ had received $\geq$ two prior systemic therapies. At median follow-up of 13.4 months, investigator-assessed ORR was $55 \%(95 \% \mathrm{Cl}, 45.2$ to 63.8), and disease control rate for $\geq 12$ weeks was $80 \%$. Median duration of response was not reached; most responses (94\%) were ongoing at data cutoff. Progression-free survival rates were 76\% (9 months) and $71 \%$ (12 months); respective OS rates were $87 \%$ and $85 \%$. Statistically significant and clinically meaningful improvements were observed in patient-reported outcomes, including functioning, symptoms, and quality of life. Grade 3 to 4 treatment-related adverse events (AEs) occurred in 32\% of patients and were manageable. Patients (13\%) who discontinued treatment because of study drug-related AEs had an ORR (63\%) consistent with that of the overall population.

\section{Conclusion}

Nivolumab plus ipilimumab demonstrated high response rates, encouraging progression-free survival and OS at 12 months, manageable safety, and meaningful improvements in key patientreported outcomes. Indirect comparisons suggest combination therapy provides improved efficacy relative to anti-programmed death-1 monotherapy and has a favorable benefit-risk profile. Nivolumab plus ipilimumab provides a promising new treatment option for patients with dMMR/MSI-H mCRC.

\section{J Clin Oncol 36:773-779. (C) 2018 by American Society of Clinical Oncology}

\section{INTRODUCTION}

Colorectal cancer (CRC) remains a leading cause of cancer-related death worldwide, with a 5-year survival rate of $14 \%$ in patients with metastatic CRC (mCRC). ${ }^{1,2}$ Patients with DNA mismatch repairdeficient (dMMR)/microsatellite instability-high (MSI-H) mCRC (approximately $4 \%$ of patients) ${ }^{3-5}$ are a distinct biomarker-defined population that benefits less from conventional chemotherapy; evolving data show poorer outcomes in key clinical parameters in these patients compared with those with MMR-proficient/microsatellite stable mCRC. ${ }^{4-9}$

Evidence from recent studies of antiprogrammed death-1 (PD-1) checkpoint inhibitors has demonstrated that dMMR/MSI-H status is a biomarker predictive of response to 
anti-PD-1 therapy ${ }^{3,10-13}$; thus, universal dMMR/MSI-H testing is recommended for patients with mCRC. ${ }^{14-16}$ In the monotherapy cohort of CheckMate-142, nivolumab, the fully human immunoglobulin G4 monoclonal antibody inhibitor of PD-1, provided durable responses (investigator-assessed objective response rate [ORR], 31\%; median duration of response [DOR], not yet reached with median follow-up of 12.0 months), sustained disease control (disease control rate $[\mathrm{DCR}] \geq 12$ weeks, 69\%), progression-free survival (PFS) rates of 54\% (9 months) and 50\% (12 months), and overall survival (OS) rates of $78 \%$ (9 months) and $73 \%$ (12 months) in previously treated patients with dMMR/MSI-H mCRC. ${ }^{11}$ Nivolumab is approved in the United States for the treatment of adult and pediatric (age $\geq 12$ years) patients with $\mathrm{dMMR} / \mathrm{MSI}-\mathrm{H}$ mCRC who had disease progression after treatment with a fluoropyrimidine, oxaliplatin, and irinotecan. ${ }^{17}$ Ipilimumab is a fully human immunoglobulin G1 monoclonal antibody that targets the cytotoxic T-cell lymphocyte antigen-4 (CTLA-4) checkpoint receptor. ${ }^{18}$ In preclinical and clinical settings, the combination of nivolumab plus ipilimumab has provided enhanced activity over nivolumab monotherapy, ${ }^{19-21}$ and the combination is approved for the treatment of metastatic melanoma using specific dosing (nivolumab $1 \mathrm{mg} / \mathrm{kg}$ plus ipilimumab $3 \mathrm{mg} / \mathrm{kg}$ once every 3 weeks for four doses followed by nivolumab $3 \mathrm{mg} / \mathrm{kg}$ once every 2 weeks). ${ }^{17}$

Presented here are efficacy, safety, biomarker, and patientreported outcome (PRO) analyses from the complete population of patients in the nivolumab plus ipilimumab cohort of CheckMate142 , which, to our knowledge, is the largest single-study report of combination immunotherapies in patients with dMMR/MSI-H mCRC.

\section{PATIENTS AND METHODS}

\section{Study Design and Participants}

CheckMate-142 is an ongoing, multicenter, open-label, phase II trial. Patients in the nivolumab plus ipilimumab cohort were treated at 28 sites in eight countries. Eligible patients were age $\geq 18$ years and had histologically confirmed recurrent CRC or mCRC assessed as dMMR and/or MSI-H per local guidelines. Patients had disease progression on or after or were intolerant of $\geq$ one prior systemic treatment that included a fluoropyrimidine and oxaliplatin or irinotecan; however, patients who refused chemotherapy were eligible. Any chemotherapy, curative-intent radiotherapy, or biologic or investigational therapy must have been completed $>28$ days before treatment initiation; focal palliative radiotherapy must have been completed $\geq 2$ weeks before starting treatment. Eligible patients had an Eastern Cooperative Oncology Group performance status of $\leq 1$ and measurable disease per Response Evaluation Criteria in Solid Tumors (RECIST; version 1.1). ${ }^{22,23}$ Patients were excluded for active, known, or suspected autoimmune disease; conditions requiring corticosteroids (prednisone equivalents $>10 \mathrm{mg}$ per day) or other immunosuppressive medication $\leq 14$ days before starting treatment; other serious or uncontrolled medical disorders; active brain or leptomeningeal metastases; or prior malignancy within the previous 3 years except for cured select localized cancers. Additional exclusion criteria included prior treatment with an anti-PD-1, anti-programmed death-ligand 1/2 (PD-L1/ PD-L2), anti-CTLA-4, or other agent targeting T-cell costimulation or immune checkpoint pathways.

Patients received nivolumab $3 \mathrm{mg} / \mathrm{kg}$ (60-minute intravenous [IV] infusion) and ipilimumab $1 \mathrm{mg} / \mathrm{kg}$ (90-minute IV infusion) once every 3 weeks for four doses and then nivolumab $3 \mathrm{mg} / \mathrm{kg}$ IV once every 2 weeks
(Appendix Fig A1, online only) until disease progression, discontinuation because of toxicity, death, withdrawal of consent, or study end. Dose modifications were not permitted. Dose interruptions for treatmentrelated adverse events (TRAEs) were allowed (Appendix, online only). Treatment beyond initial progression was permitted if the patient tolerated and benefited from study treatment per investigator assessment.

Study protocol and amendments were approved by the institutional review board or independent ethics committee at each participating center. CheckMate-142 was conducted in accordance with the Declaration of Helsinki and Good Clinical Practice guidelines, and patients provided written informed consent before enrollment.

\section{Outcomes}

The primary end point was investigator-assessed ORR (patients with best response of complete response [CR] or partial response [PR] divided by the number of treated patients) per RECIST (version 1.1). Secondary end points included ORR per blinded independent central review (BICR) and DCR (patients with best response of CR, PR, or stable disease for $\geq 12$ weeks divided by the number of treated patients). Other end points included safety and tolerability, PFS (time from first dose to first documented progression or death resulting from any cause, whichever occurred first) per investigator assessment and BICR, OS (time from the first dose to death), association between biomarker expression and efficacy, and changes from baseline in PROs.

\section{Assessments}

Tumors were assessed using computed tomography or magnetic resonance imaging per RECIST (version 1.1) $\leq 28$ days before the first dose (baseline), followed by every 6 weeks for 24 weeks and every 12 weeks thereafter until the time of disease progression or discontinuation. All responses had to be confirmed by another scan $\geq 4$ weeks later. Safety was assessed per Common Terminology Criteria for Adverse Events (version 4.0) continuously throughout treatment and for $\geq 100$ days after treatment discontinuation. ${ }^{24}$ Patients were then observed for survival every 3 months. PRO analyses were performed before the first dose of study treatment and every 6 weeks thereafter using the European Organisation for Research and Treatment of Cancer (EORTC) Core Quality of Life Questionnaire (QLQ-C30) and three-level five-dimensional EuroQol instrument (EQ-5D). ${ }^{25,26}$ EORTC QLQ-C30 assesses symptoms, functioning, and quality of life (QOL) using scales from 0 to 100 , with higher scores indicating better functioning and QOL or worse symptoms. For each scale, $\mathrm{a} \geq 10$-point change from baseline was regarded as clinically meaningful. ${ }^{27}$ EQ-5D assesses problems (none, some, or extreme) in five health dimensions (mobility, self-care, usual activities, pain or discomfort, and anxiety or depression). EQ-5D also has a visual analog scale, which allows patients to rate their health; scores range from 0 to 100 (higher values indicate better perceived health), and changes from baseline of $\geq 7$ points were deemed clinically meaningful. ${ }^{28}$

Tumor MMR and/or MSI status was evaluated before screening per local guidelines using immunohistochemistry and/or polymerase chain reaction (PCR). Samples with loss of expression of $\geq$ one mismatch repair protein per immunohistochemistry were identified as dMMR. Tumor samples were identified as MSI-H using PCR if instability was found in $\geq$ two markers when five loci were tested, in $\geq$ three of four markers when one PCR failed, or $\geq 30 \%$ of markers when $>$ five loci were tested. Additional MMR/MSI testing criteria are provided in the Appendix. Tumor PD-L1 expression ( $\geq 1 \%$ or $<1 \%$ ) was determined using archival or pretreatment biopsy tissue with the Dako 28-8 pharmDx immunohistochemistry assay (Dako North America, Carpinteria, CA). Positive PD-L1 staining was defined as complete circumferential or partial linear plasma membrane staining. BRAF/KRAS mutation status was determined at the time of screening per local guidelines. Lynch syndrome status was characterized as positive or negative by investigators based on medical history collected from clinical records; genetic testing for Lynch syndrome was not mandated in the protocol. 


\section{Statistical Analyses}

Patients were enrolled using a Simon two-stage study design. Per the protocol, only patients confirmed as MSI-H by a central laboratory were used to determine the number of responders necessary to progress from stage one to stage two. If $\leq$ six of the first 19 patients confirmed as MSI-H by a central laboratory had an objective response (CR or PR) in stage one, enrollment would end; however, if $\geq$ seven of these patients had a response in stage one, additional patients would be enrolled in stage two. Efficacy and safety were analyzed in all patients (dMMR/MSI-H per local laboratory) who received $\geq$ one dose of study treatment. Response-evaluable patients had baseline and $\geq$ one on-study tumor assessment. The $95 \% \mathrm{CI}$ for ORR was estimated using the Clopper and Pearson method. The Kaplan-Meier product-limit method was used to determine medians for DOR, PFS, and OS; corresponding 95\% CIs were calculated based on log$\log$ transformation. Descriptive statistics were used to characterize patient characteristics, PRO analyses, and safety. Missing PRO data were treated as indicated by the scoring manuals. ${ }^{25}$ For inferential PRO analyses, changes in mean scores over time were analyzed using linear mixed models adjusted for baseline score. ${ }^{29}$ Statistical analyses were performed using SAS software (version 9.02; SAS Institute, Cary, NC).

\section{RESULTS}

\section{Baseline Characteristics and Patient Disposition}

Patients were enrolled in the nivolumab plus ipilimumab combination cohort of CheckMate-142 from May 2015 through September 2016. Twenty-seven patients were enrolled in stage one, of whom 19 were confirmed as MSI-H per central laboratory. A sufficient number of confirmed investigator-assessed responses were reported in these 19 patients, and additional patients $(n=92)$ were enrolled in stage two per the protocol. At data cutoff (July 2017), 119 patients with dMMR/MSI-H mCRC were treated in stages one and two. Median duration of follow-up (potential time on study from first dose to data cutoff) was 13.4 months (range, 9 to 25 months). Most patients $(68 \%)$ were age $<65$ years, and $76 \%$ had received $\geq$ two prior lines of systemic therapy (Table 1 ); $69 \%$ of patients received prior chemotherapy with oxaliplatin, irinotecan, and a fluoropyrimidine. BRAF and KRAS mutations were identified in $24 \%$ and $37 \%$ of patients, respectively.

Most patients $(n=75 ; 63 \%)$ were still receiving treatment at data cutoff. Among patients $(\mathrm{n}=44)$ who discontinued therapy, the primary reasons were disease progression $(n=23 ; 19 \%)$, AEs related to study drug $(\mathrm{n}=16 ; 13 \%)$, and AEs unrelated to study drug $(\mathrm{n}=2 ; 2 \%)$; additional reasons included loss to follow-up, death, and patient did not present for restaging (each $n=1 ; 1 \%$ ). A median of 24 doses of nivolumab (range, one to 55 doses) and four of ipilimumab (range, one to four doses) were received; $76 \%$ and $85 \%$ of patients had a relative dose intensity $\geq 90 \%$ for nivolumab and ipilimumab, respectively.

\section{Efficacy}

Of 119 patients, $54.6 \%$ (95\% CI, 45.2 to 63.8 ) achieved an objective response per investigator assessment, including 3.4\% with CRs and 51.3\% with PRs (Table 2). Disease control for $\geq 12$ weeks was achieved in $80 \%$ (95\% CI, 71.5 to 86.6) of patients. Outcomes per investigator assessment were $91 \%$ concordant with BICR results. The ORR per BICR was $49 \%$ (95\% CI, 39.5 to 58.1), including $4 \%$ of patients with CRs and $45 \%$ with PRs; DCR for

\begin{tabular}{|c|c|}
\hline Characteristic & No. (\%) \\
\hline \multicolumn{2}{|l|}{ Age, years } \\
\hline Median & 58.0 \\
\hline Range & $21-88$ \\
\hline$<65$ & $81(68)$ \\
\hline Male sex & $70(59)$ \\
\hline \multicolumn{2}{|l|}{ Race } \\
\hline White & $109(92)$ \\
\hline Black & $2(2)$ \\
\hline Asian & $3(3)$ \\
\hline Other & $5(4)$ \\
\hline \multicolumn{2}{|l|}{ ECOG performance status } \\
\hline 0 & $54(45)$ \\
\hline 1 & $65(55)$ \\
\hline \multicolumn{2}{|l|}{ Disease stage at diagnosis } \\
\hline ॥ & $14(12)$ \\
\hline III & $52(44)$ \\
\hline IV & $53(45)$ \\
\hline \multicolumn{2}{|l|}{ Primary tumor location } \\
\hline Right colon & $65(55)$ \\
\hline Left and sigmoid colon & $30(25)$ \\
\hline Transverse colon & $15(13)$ \\
\hline Rectum & $6(5)$ \\
\hline Colon, NOS & $3(3)$ \\
\hline \multicolumn{2}{|l|}{ No. of prior systemic treatments } \\
\hline 0 & $1(1)$ \\
\hline 1 & $27(23)$ \\
\hline 2 & $43(36)$ \\
\hline$\geq 3$ & $48(40)$ \\
\hline \multicolumn{2}{|l|}{ Prior therapies received } \\
\hline Fluoropyrimidines (5-fluorouracil or capecitabine) & 118 (99) \\
\hline Oxaliplatin & 111 (93) \\
\hline Irinotecan & $87(73)$ \\
\hline VEGF inhibitors* & $68(57)$ \\
\hline EGFR inhibitors ${ }^{\dagger}$ & $35(29)$ \\
\hline Regorafenib & $11(9)$ \\
\hline Trifluridine/tipiracil & $2(2)$ \\
\hline Other experimental drugs & $3(3)$ \\
\hline Other chemotherapy & $8(7)$ \\
\hline Prior radiotherapy & $20(17)$ \\
\hline \multicolumn{2}{|l|}{ Mutation status } \\
\hline BRAFIKRAS wild type & $31(26)$ \\
\hline BRAF mutation & $29(24)$ \\
\hline KRAS mutation & $44(37)$ \\
\hline Unknown & $15(13)$ \\
\hline \multicolumn{2}{|l|}{ Tumor PD-L1 expression quantifiable at baseline } \\
\hline$\geq 1 \%$ & $26(22)$ \\
\hline$<1 \%$ & $65(55)$ \\
\hline Unknown & $28(24)$ \\
\hline \multicolumn{2}{|l|}{ Clinical history of Lynch syndromeł } \\
\hline Yes & 35 (29) \\
\hline No & $31(26)$ \\
\hline Unknown & $53(45)$ \\
\hline
\end{tabular}

Abbreviations: ECOG, Eastern Cooperative Oncology Group; EGFR, epidermal growth factor receptor; NOS, not otherwise specified; PD-L1, programmed death-ligand 1; VEGF, vascular endothelial growth factor.

*VEGF inhibitors included bevacizumab, aflibercept, and ramucirumab.

tEGFR inhibitors included cetuximab and panitumumab.

¥Lynch syndrome designation was based on the clinical records of patients at sites in countries where this reporting was permitted (excluded Italy).

$\geq 12$ weeks was observed in $79 \%$ (95\% CI, 70.6 to 85.9) of patients (Appendix Table A1, online only). Additional efficacy outcomes per BICR are presented in Appendix Figures A2 and A3 (online only). Investigator-assessed responses were observed irrespective of tumor BRAF or KRAS mutation status, tumor PD-L1 expression, 


\begin{tabular}{lcr}
\hline \multicolumn{2}{|c|}{$\begin{array}{c}\text { Table 2. ORR, Best Overall Response, and DCR per Investigator Assessment } \\
(\mathrm{N}=119)\end{array}$} \\
\hline \multicolumn{1}{c}{ Response } & No. (\%) & $95 \% \mathrm{Cl}$ \\
\hline ORR & $65(55)$ & 45.2 to 63.8 \\
Best overall response & & \\
Complete response & $4(3)$ & \\
Partial response & $61(51)$ & \\
Stable disease & $37(31)$ & \\
Progressive disease & $14(12)$ & \\
Not determined & $3(3)$ & 71.5 to 86.6 \\
Disease control for $\geq 12$ weeks & $95(80)$ &
\end{tabular}

Abbreviations: $\mathrm{DCR}$, disease control rate; $\mathrm{ORR}$, objective response rate.

or clinical history of Lynch syndrome (Appendix Table A2, online only). The ORR and DCR in patients with a BRAF mutation were $55 \%$ and $79 \%$, respectively.

Among evaluable patients $(\mathrm{n}=115), 78 \%$ had a reduction in tumor burden from baseline per investigator assessment (Fig 1A). Median time to response was 2.8 months (range, 1 to 14 months). Responses were durable, with $94 \%$ of responders having ongoing responses at data cutoff, and $83 \%$ had responses lasting $\geq 6$ months (Fig 1B). The median DOR was not reached (95\% CI, not estimable). Median PFS per investigator assessment was not reached after 33 PFS events; 9- and 12-month PFS rates were 76\% ( $95 \%$ CI, 67.0 to 82.7 ) and $71 \%$ (95\% CI, 61.4 to 78.7 ), respectively (Fig 2A). The median OS was not reached (95\% CI, not estimable), and the 9 - and 12 -month OS rates were $87 \%$ (95\% CI, 80.0 to 92.2$)$ and $85 \%$ (95\% CI, 77.0 to 90.2 ), respectively (Fig $2 \mathrm{~B}$ ).

\section{PROs}

PRO questionnaire completion rates ranged from $80 \%$ to $100 \%$ through week 91 , after which $<10$ patients were eligible for on-treatment assessment (Appendix Table A3, online only). While on study, most patients $(\geq 60 \%)$ maintained functioning and global health status/QOL without worsening of symptoms per EORTC QLQ-C30 (Appendix Table A4, online only). After adjustment for baseline score, statistically significant and clinically meaningful improvements (mean change from baseline $\geq 10$ points) were reported in key PROs, including symptoms, functioning, and global health status/QOL by week 13 or earlier, and these improvements were largely maintained in patients receiving treatment (Appendix Fig A4A to Fig A4H, online only). Although not demonstrating a mean change from baseline $\geq 10$ points at most on-treatment time points, statistically significant improvements were reported for nausea or vomiting, dyspnea, diarrhea, cognitive functioning, and physical functioning (Appendix Fig A4I to Fig A4M). Per EQ-5D, 6\% (self-care) to 63\% (pain) of patients reported health problems at baseline; notable $(>10 \%)$ reductions in patients reporting health problems were observed as early as week 13 for all dimensions (Appendix Table A5, online only). After adjustment for baseline score, statistically significant and clinically meaningful improvements in the visual analog scale of EQ-5D were observed by week 19, and these improvements were maintained in patients continuing treatment (Appendix Fig A5, online only).

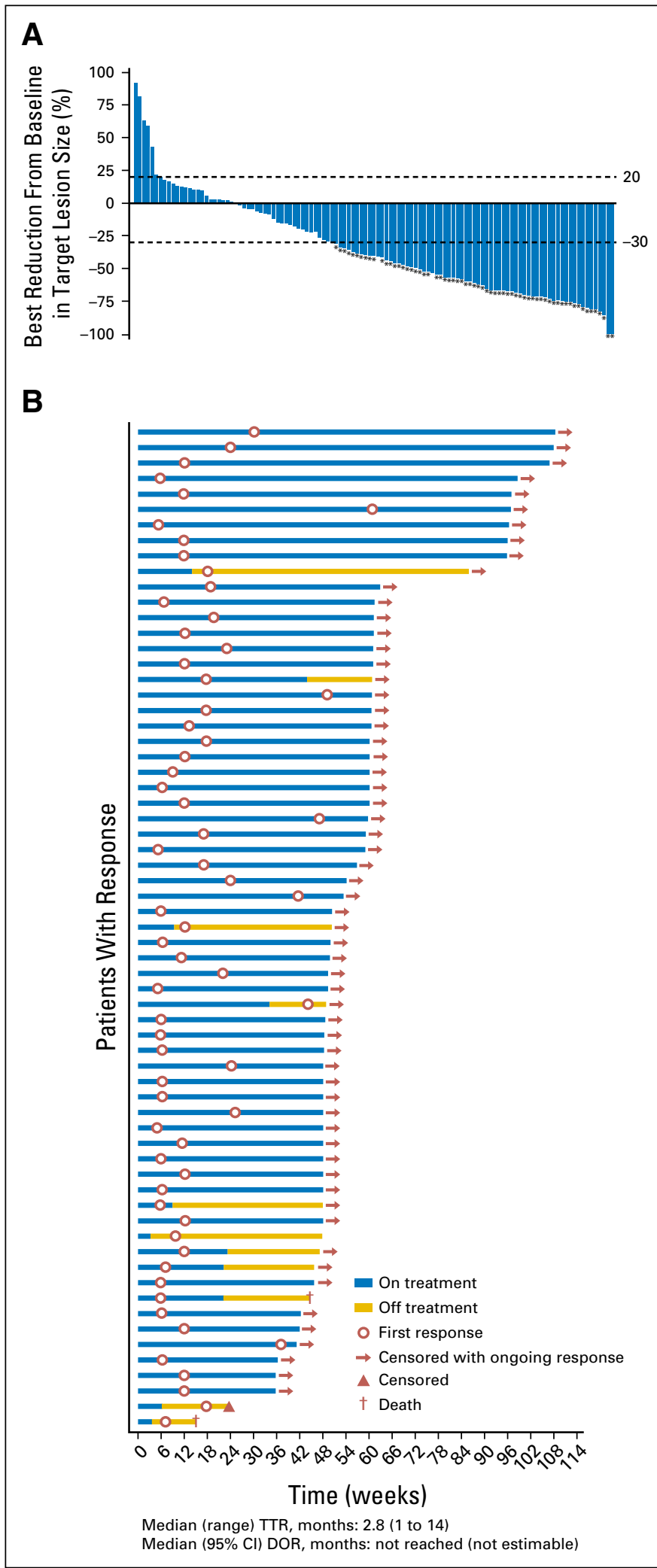

Fig 1. (A) Waterfall plot depicting the best change from baseline in target lesion size per investigator assessment. (B) Characteristics of patients with a response per investigator assessment. Bars indicate the duration of progression-free survival (PFS). DOR, duration of response; TTR, time to response. $\left({ }^{*}\right)$ Patient with confirmed response. 


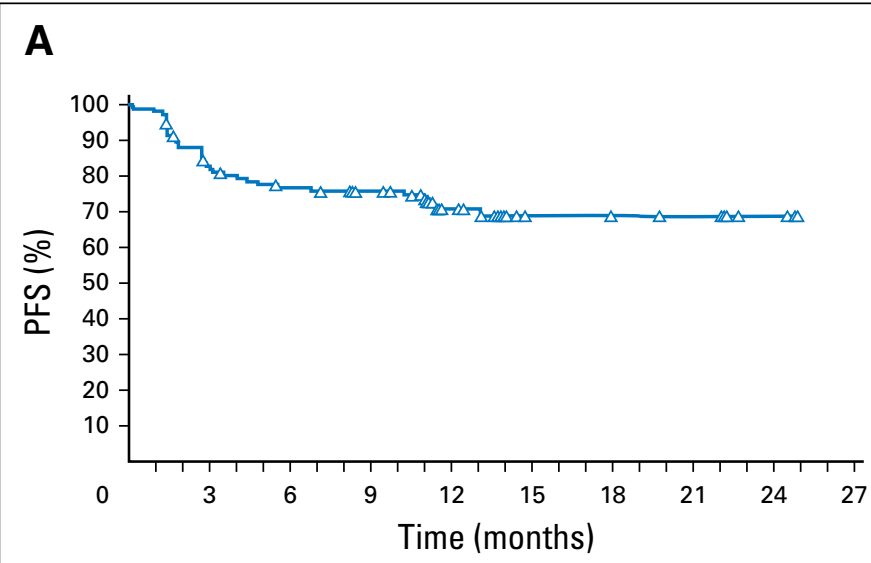

No. at risk:

$\begin{array}{llllllllll}119 & 95 & 86 & 78 & 39 & 12 & 11 & 10 & 3 & 0\end{array}$
B

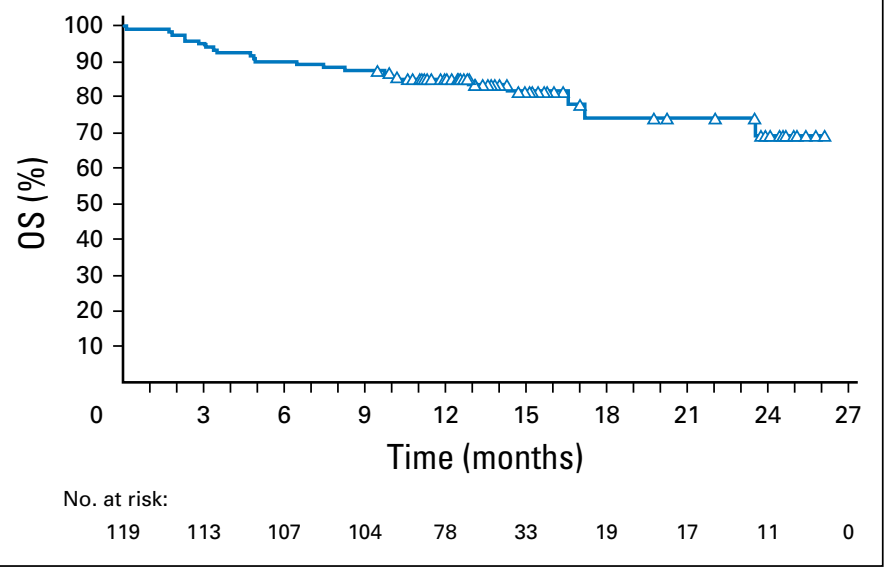

Fig 2. Kaplan-Meier plots of (A) progression-free survival (PFS) per investigator assessment and (B) overall survival (OS) in all patients.

\section{Safety}

Any-grade TRAEs were reported in $73 \%$ of patients, with the most common being diarrhea (22\%), fatigue $(18 \%)$, and pruritus (17\%; Table 3). Thirty-two percent of patients experienced a grade $3(27 \%)$ or $4(5 \%)$ TRAE, with elevated AST and/or ALT $(11 \%)$, elevated lipase (4\%), anemia (3\%), and colitis (3\%) occurring in > two patients. Serious TRAEs were reported in 23\% (any grade) and $20 \%$ (grade 3 to 4 ) of patients. TRAEs led to discontinuation in $13 \%$ (any grade) and 10\% (grade 3 to 4 ) of patients; the only events leading to discontinuation in $>$ one patient were autoimmune hepatitis and acute kidney injury $(2 \%$ each $)$. Among patients $(n=16)$ whose primary reason for discontinuing treatment was an $\mathrm{AE}$ related to study drug, the ORR was $63 \%$, DCR for $\geq 12$ weeks was $81 \%$, and median DOR was not reached, consistent with efficacy results in the overall population. Any-grade select TRAEs (events with potential immunologic etiology) analyzed by organ category occurred in 29\% (skin), 25\% (endocrine), 23\% (GI), 19\% (hepatic), and $5 \%$ (pulmonary, renal) of patients; the median time to

Table 3. Summary of TRAEs With Nivolumab in Combination With Ipilimumab $(\mathrm{N}=119)$

\begin{tabular}{lccc}
\hline & \multicolumn{3}{c}{ No. (\%) } \\
\cline { 2 - 4 } \multicolumn{1}{c}{ TRAE } & Grade 1-2 & Grade 3 & Grade 4 \\
\hline Any TRAE & $49(41)$ & $32(27)$ & $6(5)$ \\
Diarrhea* & $24(20)$ & $2(2)$ & 0 \\
Fatigue* $^{*}$ & $19(16)$ & $2(2)$ & 0 \\
Pruritus* $^{*}$ & $18(15)$ & $2(2)$ & 0 \\
Pyrexia* $_{\text {Increased AST* }}$ & $18(15)$ & 0 & 0 \\
Hypothyroidism* $^{*}$ & $8(7)$ & $9(8)$ & 0 \\
Nausea* $_{\text {Increased ALT* }}^{*}$ & $15(13)$ & $1(1)$ & 0 \\
Rash* & $14(12)$ & $1(1)$ & 0 \\
Hyperthyroidism* & $6(5)$ & $8(7)$ & 0 \\
\hline
\end{tabular}

NOTE. TRAEs were assessed during treatment and for up to 30 days after the last dose of study treatment according to National Cancer Institute Common Terminology Criteria for Adverse Events (version 4.0).

Abbreviation: ALT, alanine aminotransferase; AST, aspartate aminotransferase;

TRAE, treatment-related adverse event.

${ }^{*}$ Reported in $>10 \%$ of patients. onset ranged from 5.2 to 12.6 weeks (Appendix Table A6, online only). Some patients (range, $22 \%$ to $56 \%$ ) received immunemodulating medication to manage their select TRAEs. With the use of protocol-specified management algorithms, select TRAEs resolved in most patients (range, 71\% to 96\%), except for endocrine TRAEs, which resolved in $40 \%$ of patients. The median time to resolution of nonendocrine select TRAEs ranged from 1.5 to 9.0 weeks; the median time to resolution of endocrine TRAEs was not reached. No treatment-related deaths were reported.

\section{DISCUSSION}

Although the development of PD-1 inhibitors provided an important advance in the treatment of patients with $\mathrm{dMMR} / \mathrm{MSI}-\mathrm{H}$ mCRC, ${ }^{3,10-12}$ an opportunity remains to explore rational combinations to further improve these results. Nivolumab and ipilimumab act synergistically to promote T-cell antitumor activity through complementary mechanisms of action. ${ }^{17,18,20,21}$ Results of the nivolumab plus ipilimumab cohort reported here demonstrate a manageable safety profile and robust clinical activity, with an ORR of $55 \%$, DCR for $\geq 12$ weeks of $80 \%$, PFS rates of $76 \%$ (9 months) and $71 \%$ (12 months), and OS rates of $87 \%$ ( 9 months) and $85 \%$ (12 months); responses in patients with dMMR/MSI-H mCRC were observed irrespective of tumor PD-L1 expression, $B R A F / K R A S$ mutation status, or clinical history of Lynch syndrome. The favorable benefit-risk profile seen in this cohort suggests a role for combination checkpoint inhibitor therapy in the treatment of patients with dMMR/MSI-H mCRC.

In CheckMate-142, monotherapy and combination therapy cohorts were neither randomly assigned nor designed for formal comparison; however, considering the limitations of an indirect comparison, nivolumab plus ipilimumab provided a numerically higher response rate $(55 \% ; 95 \% \mathrm{CI}, 45$ to 64$)$ relative to the response rate (31\%; 95\% CI, 21 to 43 ) with nivolumab monotherapy in a similar population of patients $(n=74)$ with a comparable median follow-up time. ${ }^{11}$ Likewise, with the limitations of crosstrial comparisons in mind, the response rate with nivolumab plus ipilimumab in CheckMate-142 was numerically higher relative to 


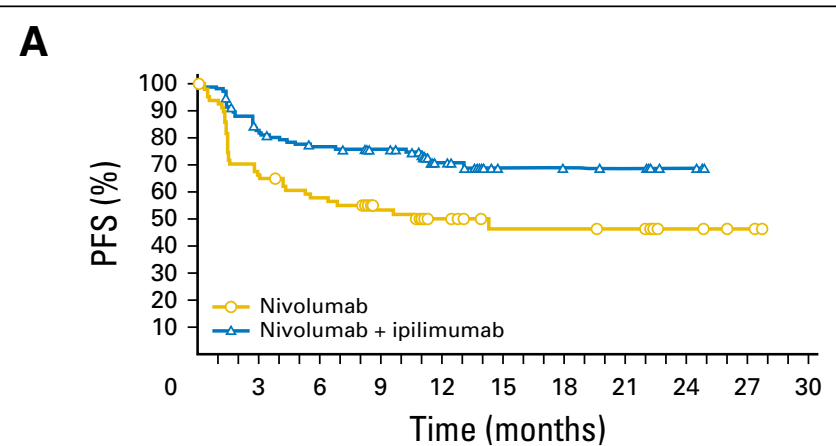

No. at risk: $\begin{array}{llllllllllll}\text { Nivolumab } & 74 & 48 & 41 & 32 & 17 & 12 & 12 & 11 & 6 & 3 & 0\end{array}$ $\begin{array}{llllllllllll}\text { Nivolumab }+ & 119 & 95 & 86 & 78 & 39 & 12 & 11 & 10 & 3 & 0 & 0\end{array}$ ipilimumab
B

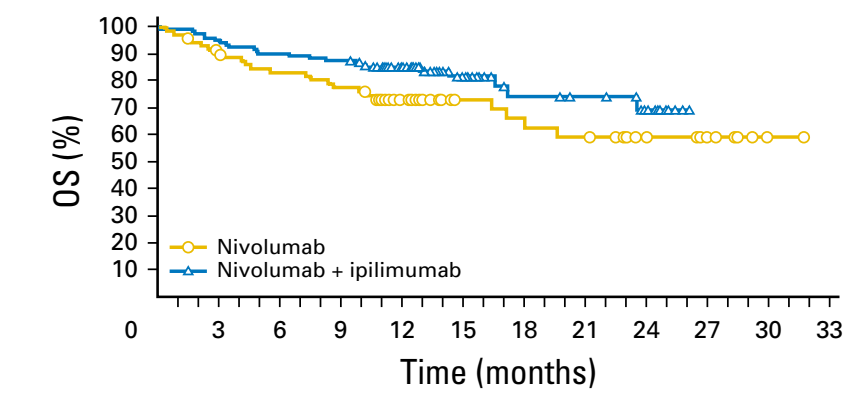

No. at risk:

$\begin{array}{lcccccccccccc}\text { Nivolumab } & 74 & 64 & 59 & 55 & 37 & 21 & 19 & 17 & 11 & 6 & 1 & 0 \\ \text { Nivolumab + } & 119 & 113 & 107 & 104 & 78 & 33 & 19 & 17 & 11 & 0 & 0 & 0\end{array}$ ipilimumab

Fig 3. Kaplan-Meier plots of (A) progression-free survival (PFS) per investigator assessment and (B) overall survival (OS) in patients treated with nivolumab plus ipi-

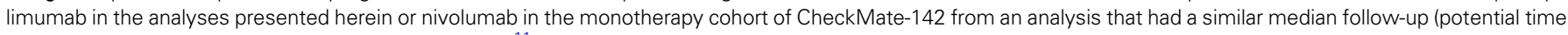
on study from first dose to data cutoff: 13.4 months). ${ }^{11}$

the rate $(28 \% ; 95 \% \mathrm{CI}, 17$ to 41$)$ reported with pembrolizumab monotherapy in previously treated patients $(n=61)$ with MSI-H mCRC in KEYNOTE-164, of whom $15 \%$ had BRAF mutations and $90 \%$ had received $\geq$ two prior lines of therapy. ${ }^{12,13}$ Although results with pembrolizumab monotherapy in KEYNOTE-016 showed numerically higher response rates (57\%; 95\% CI, 39 to $73)$, the limited number of patients $(n=28)$ and sites $(n=6$; United States only) as well as a high rate of Lynch syndrome (54\%) may have biased findings in this study. ${ }^{10,30,31}$ In an indirect comparison of PFS and OS, estimated 12-month PFS (71\%) and OS $(85 \%)$ rates with nivolumab plus ipilimumab were numerically higher relative to those observed with anti-PD-1 monotherapies (nivolumab [CheckMate-142]: PFS, 50\% and OS, 73\%; pembrolizumab [KEYNOTE-164]: PFS, 34\% and OS, 72\%). ${ }^{11-13}$ Despite the caveats associated with indirect comparisons, KaplanMeier plots of PFS and OS with nivolumab monotherapy and nivolumab plus ipilimumab combination therapy in CheckMate142 suggest that the addition of ipilimumab may improve the longterm clinical benefit of nivolumab (Fig 3) ${ }^{11}$; additional investigation is warranted.

Nivolumab plus ipilimumab combination therapy has been investigated using different doses and schedules in other tumor types, and the safety profile has been found to be influenced by the ipilimumab dose..$^{20,21,32} \mathrm{In} \mathrm{mCRC}$, nivolumab $3 \mathrm{mg} / \mathrm{kg}$ plus ipilimumab $1 \mathrm{mg} / \mathrm{kg}$ once every 3 weeks was selected based on the safety cohort of CheckMate-142. ${ }^{33}$ At this dose, a majority of patients received all four doses of ipilimumab, and the safety profile was manageable. Importantly, the incidence of diarrhea and colitis with combination therapy did not seem elevated in this population compared with that in patients with other solid tumors. $^{20,21,32}$ The rate of any-grade and grade 3 to 4 TRAEs was $73 \%$ and $32 \%$, respectively, and efficacy was maintained (ORR, $63 \%$; DCR, $81 \%$ ) in patients who discontinued treatment because of study drug-related AEs. ${ }^{11}$ Nonendocrine select TRAEs resolved in most patients (range, $71 \%$ to $96 \%$ ) in a median of 1.5 to 9.0 weeks with the use of protocol-specified management algorithms; endocrine TRAEs resolved in $40 \%$ of patients. Of note, the overall rate of any-grade TRAEs in the combination therapy cohort was comparable to that in the nivolumab monotherapy cohort (70\%), and the rates of discontinuation because of study drug-related AEs ( $13 \%$ and $7 \%$, respectively) were modest in each cohort. ${ }^{11}$ Importantly, patients exhibited statistically significant and clinically meaningful on-treatment improvements with combination therapy in key PROs, including symptoms, functioning, and QOL.

In conclusion, the results presented here demonstrate that nivolumab in combination with ipilimumab provided durable responses, high DCR, encouraging survival rates, manageable safety, and meaningful improvements in key PROs in previously treated patients with $\mathrm{dMMR} / \mathrm{MSI}-\mathrm{H}$ mCRC. Considering the indirect comparisons that suggest numerically higher response rates and an improved long-term clinical benefit with nivolumab plus ipilimumab relative to anti-PD-1 monotherapy, and the favorable benefit-risk profile of combination therapy, nivolumab plus ipilimumab represents a promising new treatment option in these patients. Evaluation of nivolumab plus ipilimumab as a firstline therapy (phase II) in patients with dMMR/MSI-H mCRC is ongoing.

\section{AUTHORS' DISCLOSURES OF POTENTIAL CONFLICTS OF INTEREST}

Disclosures provided by the authors are available with this article at jco.org.

\section{AUTHOR CONTRIBUTIONS}

Conception and design: Michael J. Overman, Rebecca A. Moss, Scott Kopetz

Collection and assembly of data: Michael J. Overman, Sara Lonardi, Ka Yeung Mark Wong, Heinz-Josef Lenz, Fabio Gelsomino, Massimo Aglietta, Michael A. Morse, Eric Van Cutsem, Ray McDermott, Andrew Hill, Michael B. Sawyer, Alain Hendlisz, Bart Neyns, Magali Svrcek, Scott Kopetz, Thierry André

Data analysis and interpretation: All authors

Manuscript writing: All authors

Final approval of manuscript: All authors

Accountable for all aspects of the work: All authors 


\section{REFERENCES}

1. National Cancer Institute: Surveillance, Epidemiology, and End Results Program: Cancer stat facts: Colon and rectum cancer. https://seer.cancer. gov/statfacts/html/colorect.html

2. International Agency for Research on Cancer: GLOBOCAN 2012: Estimated cancer incidence, mortality and prevalence worldwide in 2012-Cancer fact sheets. http://globocan.iarc.fr/Pages/fact_sheets_cancer.aspx

3. Le DT, Durham JN, Smith KN, et al: Mismatch repair deficiency predicts response of solid tumors to PD-1 blockade. Science 357:409-413, 2017

4. Koopman M, Kortman GA, Mekenkamp L, et al: Deficient mismatch repair system in patients with sporadic advanced colorectal cancer. $\mathrm{Br} J$ Cancer 100:266-273, 2009

5. Venderbosch S, Nagtegaal ID, Maughan TS, et al: Mismatch repair status and BRAF mutation status in metastatic colorectal cancer patients: A pooled analysis of the CAIRO, CAIRO2, COIN, and FOCUS studies. Clin Cancer Res 20:5322-5330, 2014

6. Innocenti F, Ou F, Zemla T, et al: Somatic DNA mutations, MSI status, mutational load (ML): Association with overall survival (OS) in patients (pts) with metastatic colorectal cancer (mCRC) of CALGB/ SWOG 80405 (alliance). J Clin Oncol 35, 2017 (suppl; abstr 3504)

7. Stintzing $S$, Wirapati $P$, Lenz $H$, et al: Consensus molecular subgroups (CMS) of colorectal cancer (CRC) and first-line efficacy of FOLFIRI plus cetuximab or bevacizumab in the FIRE3 (AIO KRK0306) trial. J Clin Oncol 35, 2017 (suppl; abstr 3510)

8. Tougeron $D$, Cohen $R$, Sueur $B$, et al: A large retrospective multicenter study evaluating prognosis and chemosensitivity of metastatic colorectal cancer with microsatellite instability. Ann Oncol 28, 2017 (suppl 5; abstr 533P)

9. Lenz $H$, Ou F, Venook AP, et al: Impact of consensus molecular subtyping (CMS) on overall survival (OS) and progression free survival (PFS) in patients (pts) with metastatic colorectal cancer (mCRC): Analysis of CALGB/SWOG 80405 (alliance). J Clin Oncol 35, 2017 (suppl; abstr 3511)
10. Le DT, Uram JN, Wang H, et al: PD-1 blockade in tumors with mismatch-repair deficiency. N Engl J Med 372:2509-2520, 2015

11. Overman MJ, McDermott $R$, Leach JL, et al: Nivolumab in patients with metastatic DNA mismatch repair-deficient or microsatellite instability-high colorectal cancer (CheckMate 142): An open-label, multicentre, phase 2 study. Lancet Oncol 18:1182-1191, 2017

12. Diaz LA, Marabelle A, Delord J, et al: Pembrolizumab therapy for microsatellite instability high (MSI-H) colorectal cancer (CRC) and non-CRC. J Clin Oncol 35, 2017 (suppl; abstr 3071)

13. Diaz LA, Marabelle A, Kim TW, et al: Efficacy of pembrolizumab in phase 2 KEYNOTE-164 and KEYNOTE158 studies of microsatellite instability high cancers. Ann Oncol 28, 2017 (suppl 5; abstr 386P)

14. Sepulveda AR, Hamilton SR, Allegra CJ, et al: Molecular biomarkers for the evaluation of colorectal cancer: Guideline from the American Society for Clinical Pathology, College of American Pathologists, Association for Molecular Pathology, and the American Society of Clinical Oncology. J Clin Oncol 35:1453-1486, 2017

15. Van Cutsem E, Cervantes A, Adam R, et al: ESMO consensus guidelines for the management of patients with metastatic colorectal cancer. Ann Oncol 27:1386-1422, 2016

16. National Comprehensive Cancer Network: NCCN clinical practice guidelines in oncology: Colon cancer-Version 2.2017. https://www.nccn.org/ professionals/physician_gls/pdf/colon.pdf

17. Opdivo (nivolumab): US prescribing information. Bristol-Myers Squibb, Princeton, NJ, 2017

18. Yervoy (ipilimumab): US prescribing information. Bristol-Myers Squibb, Princeton, NJ, 2017

19. Curran MA, Montalvo W, Yagita $H$, et al: PD-1 and CTLA-4 combination blockade expands infiltrating $T$ cells and reduces regulatory $T$ and myeloid cells within B16 melanoma tumors. Proc Natl Acad Sci USA 107:4275-4280, 2010

20. Antonia SJ, López-Martin JA, Bendell J, et al: Nivolumab alone and nivolumab plus ipilimumab in recurrent small-cell lung cancer (CheckMate 032): A multicentre, open-label, phase 1/2 trial. Lancet Oncol 17:883-895, 2016

21. Larkin J, Chiarion-Sileni $V$, Gonzalez $R$, et al: Combined nivolumab and ipilimumab or monotherapy in untreated melanoma. N Engl J Med 373:23-34, 2015
22. Oken MM, Creech RH, Tormey DC, et al: Toxicity and response criteria of the Eastern Cooperative Oncology Group. Am J Clin Oncol 5:649-655, 1982

23. Eisenhauer EA, Therasse P, Bogaerts J, et al: New response evaluation criteria in solid tumours: Revised RECIST guideline (version 1.1). Eur J Cancer 45:228-247, 2009

24. US Department of Health and Human Services, National Cancer Institute: Common Terminology Criteria for Adverse Events (CTCAE) version 4.0. https://evs.nci.nih.gov/ftp1/CTCAE/CTCAE_4.03_ 2010-06-14_QuickReference_5x7.pdf

25. Aaronson NK, Ahmedzai S, Bergman B, et al: The European Organization for Research and Treatment of Cancer QLQ-C30: A quality-of-life instrument for use in international clinical trials in oncology. $\mathrm{J}$ Natl Cancer Inst 85:365-376, 1993

26. EuroQol Group: EuroQol: A new facility for the measurement of health-related quality of life. Health Policy 16:199-208, 1990

27. Osoba D, Rodrigues G, Myles J, et al: Interpreting the significance of changes in health-related quality-of-life scores. J Clin Oncol 16:139-144, 1998

28. Pickard AS, Neary MP, Cella D: Estimation of minimally important differences in EQ-5D utility and VAS scores in cancer. Health Qual Life Outcomes 5:70, 2007

29. Bell ML, Fairclough DL: Practical and statistical issues in missing data for longitudinal patient-reported outcomes. Stat Methods Med Res 23:440-459, 2014

30. Le DT, Uram JN, Wang $\mathrm{H}$, et al: Programmed death-1 blockade in mismatch repair deficient colorectal cancer. J Clin Oncol 34, 2016 (suppl; abstr 103)

31. Keytruda (pembrolizumab): US prescribing information. Merck, Whitehouse Station, NJ, 2017

32. Janjigian $Y Y$, Ott PA, Calvo E, et al: Nivolumab \pm ipilimumab in pts with advanced (adv)/metastatic chemotherapy-refractory (CTx-R) gastric (G), esophageal (E), or gastroesophageal junction (GEJ) cancer: CheckMate 032 study. J Clin Oncol 35, 2017 (suppl; abstr 4014)

33. Overman MJ, Kopetz $S$, Lonardi $S$, et al: Nivolumab \pm ipilimumab treatment $(t x)$ efficacy, safety, and biomarkers in patients (pts) with metastatic colorectal cancer ( $\mathrm{mCRC}$ ) with and without high microsatellite instability (MSI-H): Results from the CheckMate-142 study. Ann Oncol 27, 2016 (suppl 6; abstr 149)

\section{Affiliations}

Michael J. Overman and Scott Kopetz, University of Texas MD Anderson Cancer Center, Houston, TX; Sara Lonardi, Istituto Oncologico Veneto, Istituto di Ricovero e Cura a Carattere Scientifico, Padova; Fabio Gelsomino, University Hospital of Modena, Modena; Massimo Aglietta, Institute for Cancer Research and Treatment of Candiolo, University of Torino Medical School, Turin, Italy; Ka Yeung Mark Wong, Sydney Medical School, University of Sydney, Sydney, New South Wales; Andrew Hill, Tasman Oncology Research, Ltd., Southport, Queensland, Australia; Heinz-Josef Lenz, University of Southern California Norris Comprehensive Cancer Center, Los Angeles, CA; Michael A. Morse, Duke University Medical Center, Durham, NC; Eric Van Cutsem, University Hospitals Gasthuisberg Leuven and Katholieke Universiteit Leuven, Leuven; Alain Hendlisz, Institut Jules Bordet; Bart Neyns, Universitair Ziekenhuis Brussel, Brussels; Jean-Marie Ledeine, Bristol-Myers Squibb, Braine-L'Alleud, Belgium; Ray McDermott, St Vincent's University Hospital and Cancer Trials Ireland, Dublin, Ireland; Michael B. Sawyer, Cross Cancer Institute and University of Alberta, Edmonton, Alberta, Canada; Magali Svrcek and Thierry André, Hopital Saint Antoine, Assistance Publique Hôpitaux de Paris and Sorbonne Universités, Université Pierre et Marie Curie, Paris, France; and Rebecca A. Moss, Z. Alexander Cao, and Shital Kamble, Bristol-Myers Squibb, Princeton, NJ.

\section{Support}

Supported by Bristol-Myers Squibb, which also funded editorial assistance.

\section{Prior Presentation}

Presented in part at the 52nd Annual Meeting of the American Society of Clinical Oncology (ASCO), Chicago, IL, June 3-7, 2016; Annual Congress of the European Society of Medical Oncology (ESMO), Copenhagen, Denmark, October 7-11, 2016; 53rd ASCO Annual Meeting, Chicago, IL, June 2-6, 2017; and ESMO Annual Congress, Madrid, Spain, September 8-12, 2017. 
Durable Clinical Benefit With Nivolumab Plus Ipilimumab in DNA Mismatch Repair-Deficient/Microsatellite Instability-High Metastatic Colorectal Cancer

The following represents disclosure information provided by authors of this manuscript. All relationships are considered compensated. Relationships are self-held unless noted. I = Immediate Family Member, Inst = My Institution. Relationships may not relate to the subject matter of this manuscript. For more information about ASCO's conflict of interest policy, please refer to www.asco.org/rwc or ascopubs.org/jco/site/ifc.

Michael J. Overman

Consulting or Advisory Role: Merrimack, Bristol-Myers Squibb, Roche/ Genentech

Research Funding: Bristol-Myers Squibb, Merck, Amgen, Roche, Celgene, MedImmune

\section{Sara Lonardi}

Consulting or Advisory Role: Bayer HealthCare Pharmaceuticals, Amgen Speakers' Bureau: Roche, Eli Lilly

Research Funding: Sanofi

\section{Ka Yeung Mark Wong}

Consulting or Advisory Role: Baxalta

Speakers' Bureau: Sirtex Medical

Travel, Accommodations, Expenses: MSD Oncology

\section{Heinz-Josef Lenz}

Honoraria: Bristol-Myers Squibb, Bayer HealthCare Pharmaceuticals, Genentech, Merck Serono

Consulting or Advisory Role: Bristol-Myers Squibb, Bayer HealthCare Pharmaceuticals, Genentech, Merck Serono

Research Funding: Bristol-Myers Squibb, Roche, Genentech, Bayer HealthCare Pharmaceuticals, Taiho Pharmaceutical, Merck Serono Travel, Accommodations, Expenses: Merck Serono, Bayer HealthCare Pharmaceuticals, Boehringer Ingelheim, Bristol-Myers Squibb

\section{Fabio Gelsomino}

No relationship to disclose

\section{Massimo Aglietta}

Consulting or Advisory Role: Bristol-Myers Squibb, Merck, Roche Travel, Accommodations, Expenses: Bristol-Myers Squibb

\section{Michael A. Morse}

No relationship to disclose

\section{Eric Van Cutsem}

Consulting or Advisory Role: Bayer HealthCare Pharmaceuticals, Eli Lilly, Roche, Servier

Research Funding: Amgen (Inst), Bayer HealthCare Pharmaceuticals (Inst), Boehringer Ingelheim (Inst), Eli Lilly (Inst), Novartis (Inst), Roche (Inst), Sanofi (Inst), Celgene (Inst), Ipsen (Inst), Merck (Inst), Merck KGaA (Inst), Servier (Inst)

\section{Ray McDermott}

Honoraria: Bayer HealthCare Pharmaceuticals, Sanofi, Janssen

Pharmaceuticals, Astellas Pharma, Bristol-Myers Squibb, Merck Sharp \& Dohme, Pfizer, Novartis, Clovis Oncology

Research Funding: Sanofi (Inst), Janssen Pharmaceuticals (Inst), Bayer HealthCare Pharmaceuticals (Inst), Astellas Pharma (Inst)

Travel, Accommodations, Expenses: Pfizer, Janssen-Cilag,

\section{Andrew Hill}

Employment: Tasman Health Care PTY Ltd.

Stock or Other Ownership: Tasman Health Care PTY Ltd.

Honoraria: Bristol-Myers Squibb

Research Funding: Bristol-Myers Squibb

Travel, Accomodations, Expenses: Bristol-Myers Squibb, Merck

\section{Michael B. Sawyer}

Honoraria: Bristol-Myers Squibb, Merck, Celgene, Shire, Baxter, Nutricia Consulting or Advisory Role: Celgene, Nutricia

Research Funding: Bayer HealthCare Pharmaceuticals (Inst), Pfizer (Inst), Bristol-Myers Squibb (Inst), Corvus Pharmaceuticals (Inst), Merck (Inst) Travel, Accommodations, Expenses: Pfizer, Amgen, Novartis, Nutricia

\section{Alain Hendlisz}

No relationship to disclose

\section{Bart Neyns}

Honoraria: Bristol-Myers Squibb, Merck Sharp \& Dohme, Roche, Amgen, Novartis, Pfizer

Consulting or Advisory Role: Amgen, Pfizer, Merck, Bristol-Myers Squibb, Roche, Novartis

Speakers' Bureau: Novartis

Research Funding: Pfizer, Novartis

Travel, Accommodations, Expenses: Bristol-Myers Squibb, Merck Sharp \& Dohme, Roche, Amgen, Novartis

\section{Magali Svrcek}

Consulting or Advisory Role: Bristol-Myers Squibb

Rebecca A. Moss

Employment: Bristol-Myers Squibb

Stock or Other Ownership: Bristol-Myers Squibb

Jean-Marie Ledeine

Employment: Bristol-Myers Squibb

Stock or Other Ownership: Bristol-Myers Squibb

\section{Z. Alexander Cao}

Employment: Bristol-Myers Squibb

Stock or Other Ownership: Bristol-Myers Squibb, Novartis

\section{Shital Kamble}

Employment: Bristol-Myers Squibb

Stock or Other Ownership: Bristol-Myers Squibb

\section{Scott Kopetz}

Stock or Other Ownership: MolecularMatch, Navire

Consulting or Advisory Role: Amgen, Roche, Bayer HealthCare Pharmaceuticals, Array BioPharma, Genentech, Symphogen, EMD Serono, Merck, Karyopharm Therapeutics

Research Funding: Amgen (Inst), Sanofi (Inst), Biocartis (Inst), Guardant Health (Inst), Array BioPharma (Inst), Genentech/Roche (Inst), EMD

Serono (Inst), MedImmune (Inst), Novartis (Inst)

\section{Thierry André}

Honoraria: Roche/Genentech, Sanofi, Eli Lilly, Baxter, Bayer HealthCare Pharmaceuticals, Bristol-Myers Squibb, MSD Oncology, Boehringer Ingelheim, Celgene, Servier, Xbiotech, Novartis

Consulting or Advisory Role: Roche/Genentech, Amgen, Bristol-Myers Squibb, Mundipharma, HalioDX, MSD Oncology, Servier, Guardant Health

Speakers' Bureau: Bristol-Myers Squibb

Travel, Accommodations, Expenses: Roche/Genentech, Amgen, BristolMyers Squibb 


\section{Acknowledgment}

We thank the patients and their families, investigators, and research staff at all study sites. We also thank ONO Pharmaceutical, Osaka, Japan, and the staff of Dako North America for collaborative development of the automated programmed death-ligand 1 immunohistochemical assay. Additionally, we thank Michael Axelson, Danielle Greenawalt, David Leung, Demetrios Manekas, and Hao Tang for their support. Editorial assistance was provided by Christopher Reina of Chrysalis Medical Communications, Hamilton, NJ, and was funded by Bristol-Myers Squibb.

\section{Appendix}

\section{Dose Interruptions for Treatment-Related Adverse Events}

Dose interruptions for treatment-related adverse events (TRAEs) were allowed until resolution of these events or for $\leq 6$ weeks from the last treatment. Patients could resume treatment at the next scheduled dose after TRAE resolution; however, patients were not to skip administration of nivolumab plus ipilimumab (if toxicity allowed) to ensure that each patient received four doses of combination therapy. Patients with interruptions lasting $>6$ weeks were discontinued from the study, except when dosing was interrupted for prolonged steroid tapers to manage TRAEs or for non-TRAEs (required approval).

\section{Local Laboratory Criteria for Determining Mismatch Repair-Proficient and Microsatellite Instability Status}

DNA mismatch repair deficiency determined by immunohistochemistry refers to the loss of expression of $\geq$ one mismatch repair protein (ie, MLH1, MSH2, MSH6, or PMS2).

Microsatellite instability-high (MSI-H) is most frequently determined by polymerase chain reaction. MSI-H in tumors refers to changes in $\geq$ two of the five National Cancer Institute-recommended panels of microsatellite markers in tumor tissue. The original Bethesda guidelines proposed a panel of five microsatellite markers for the uniform analysis of MSI in Lynch syndrome (Umar A, et al: J Nat Cancer Inst 96:261-268, 2004). Individual testing sites may use a slightly different panel of markers incorporating alternative mononucleotide and/or dinucleotide markers. Regardless of the panel of markers, samples with instability in $\geq 30 \%$ of these markers are defined as MSI-H, whereas those with $<30 \%$ unstable markers are designated as MSI-low. Samples with no detectable alterations are microsatellite stable.

\section{Central Laboratory Criteria for Determining Microsatellite Instability in Combination Stage One}

In combination stage one of the two-stage Simon design, a central laboratory was used to confirm the MSI-H status of tumor tissue collected at baseline by polymerase chain reaction using modified Bethesda criteria. This panel included two mononucleotide (BAT-25 and BAT-26) and three dinucleotide (D5S346, D2S123, and D17S250) repeats (Umar A, et al: J Nat Cancer Inst 96:261$268,2004)$. Samples with instability in $\geq$ two of these markers were defined as MSI-H, whereas those with one unstable marker were designated as MSI-low. Samples with no detectable alterations were identified as microsatellite stable.

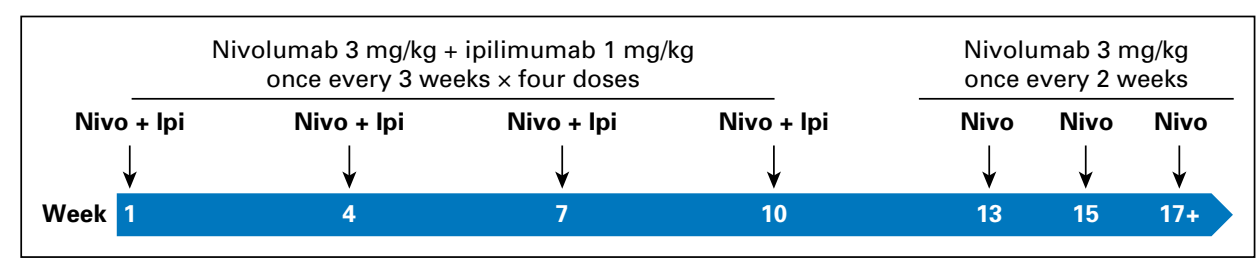

Fig A1. Nivolumab (Nivo) plus ipilimumab (Ipi) dosing schedule in CheckMate-142. 


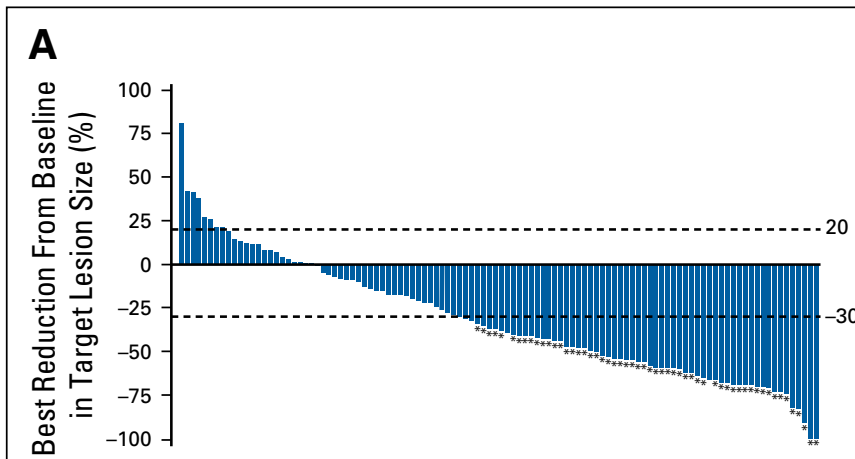

B

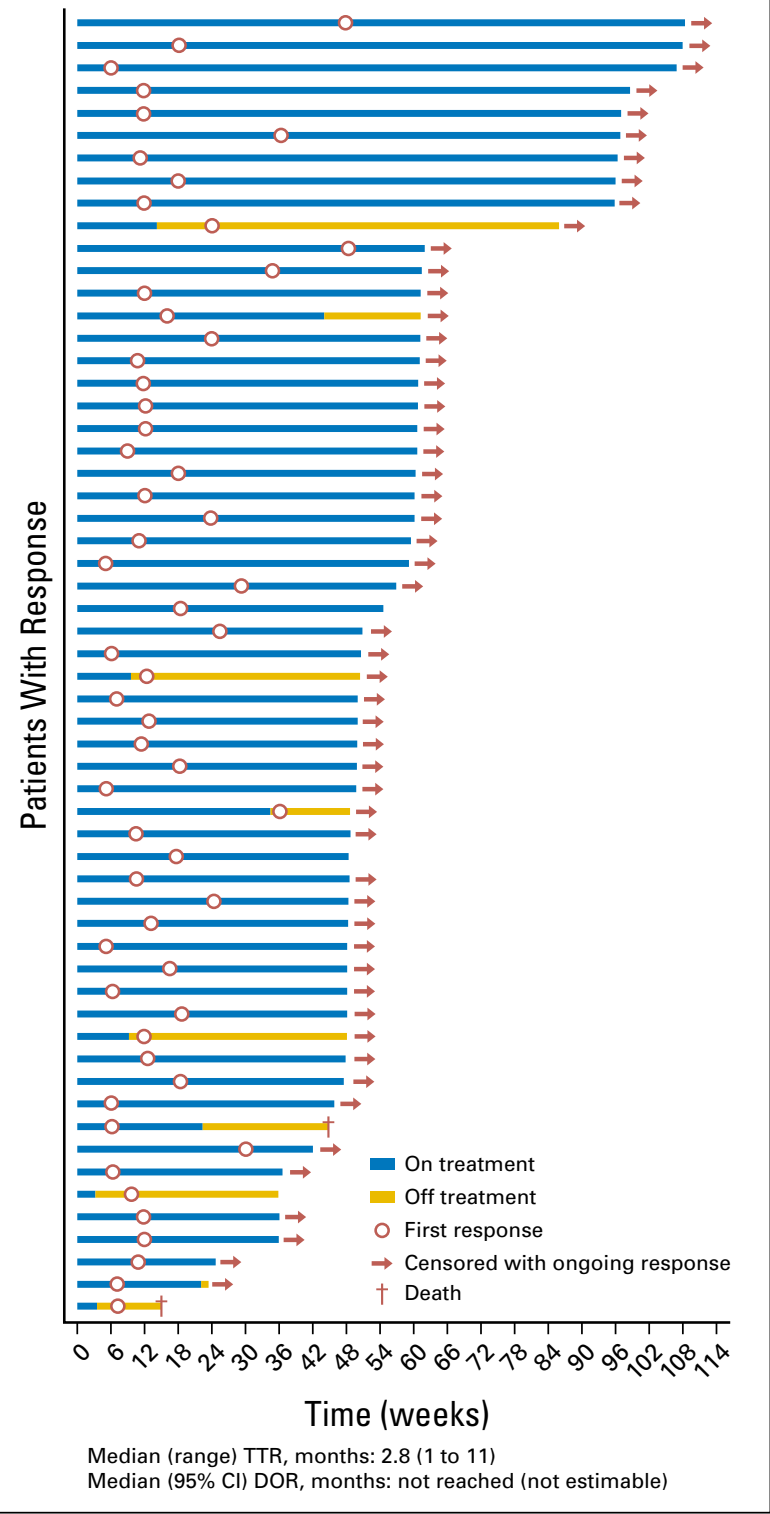

Fig A2. (A) Best reduction from baseline in target lesion size, and (B) characteristics of response per blinded independent central review. DOR, duration of response; TTR, time to response. $\left({ }^{*}\right)$ Patient with confirmed response 
Nivolumab Plus Ipilimumab in dMMR/MSI-H Metastatic CRC

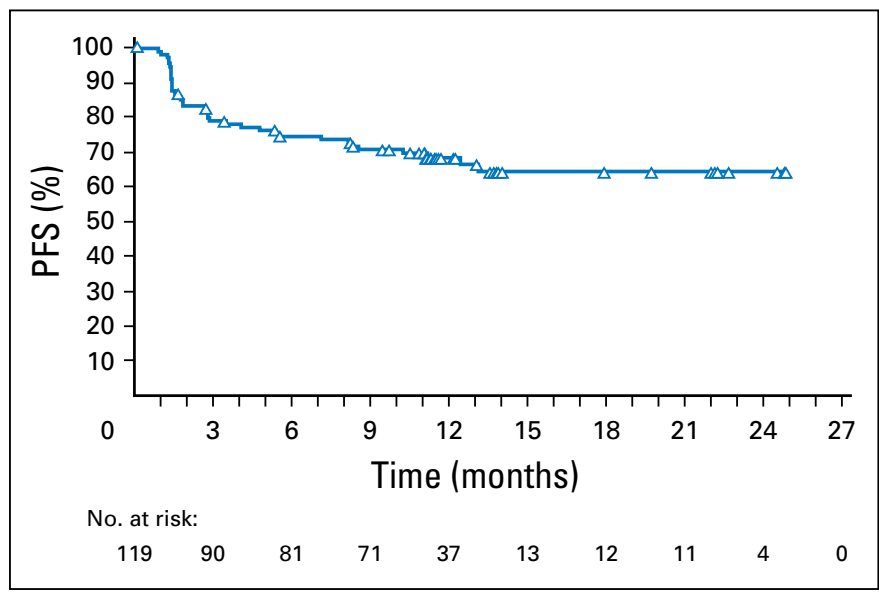

Fig A3. Progression-free survival (PFS) per blinded independent central review. 

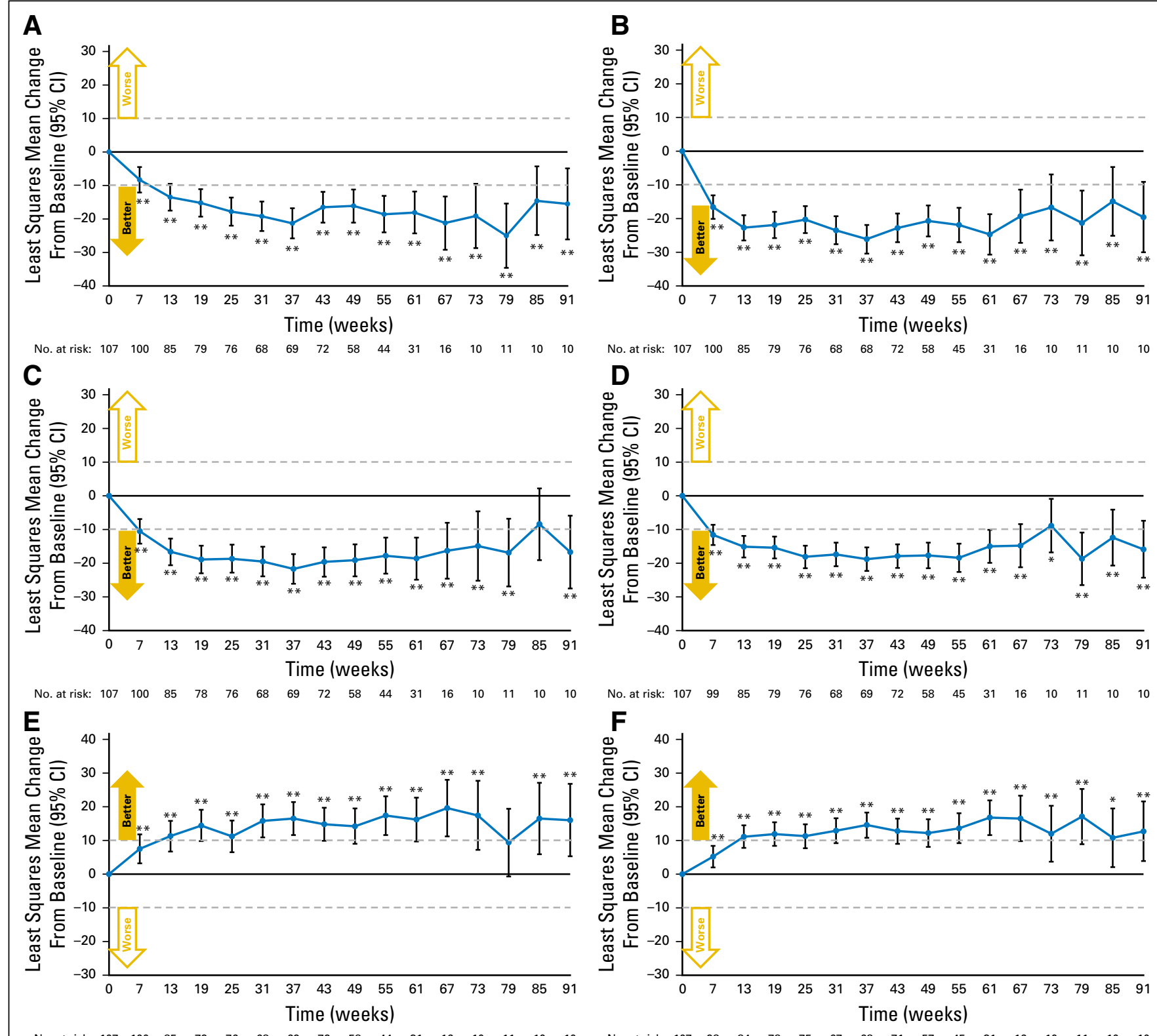

$\begin{array}{llllllllllllllllll}\text { No. at risk: } & 107 & 100 & 85 & 79 & 76 & 68 & 69 & 72 & 58 & 44 & 31 & 16 & 10 & 11 & 10 & 10 & \\ \mathbf{G} & & & & & & & & & & & & & & & & \end{array}$
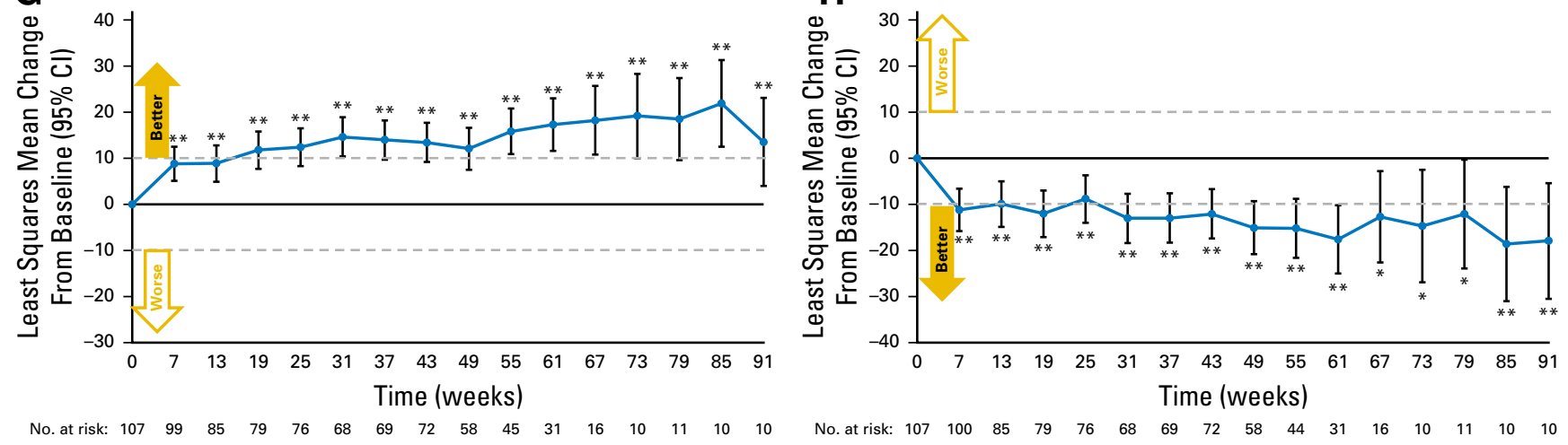

Fig A4. Patient-reported outcomes: mean change from baseline per the European Organisation for Research and Treatment of Cancer Core Quality of Life Questionnaire.

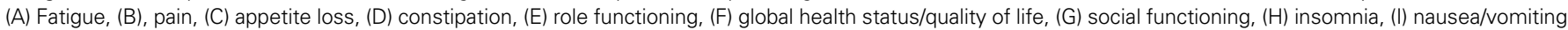
(J) dyspnea, (K) diarrhea, $(\mathrm{L})$ cognitive functioning, and $(\mathrm{M})$ physical functioning. $\left({ }^{*}\right) P<.05 .\left({ }^{*}\right) P<.01$. 


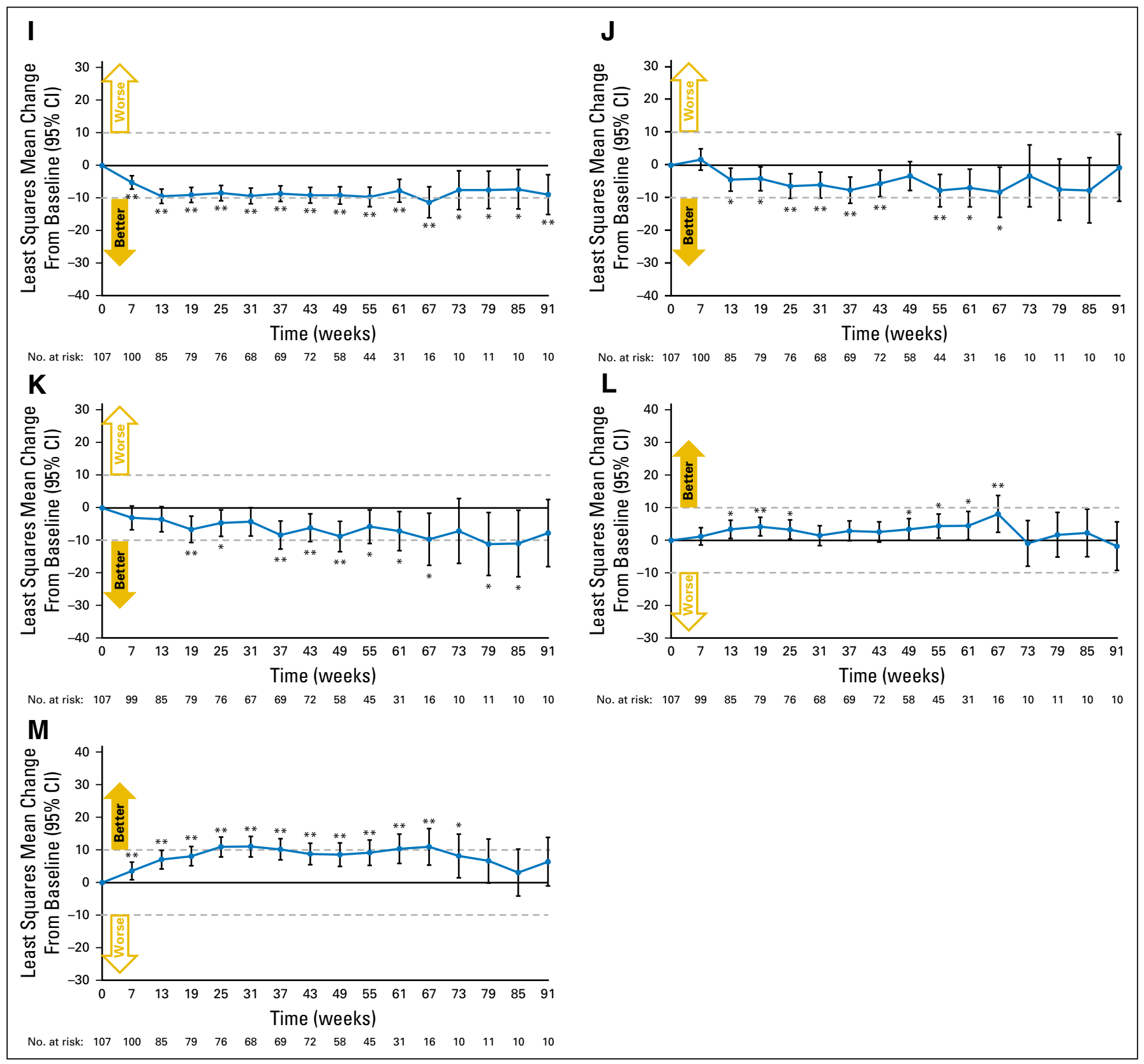

Fig A4. (Continued). 


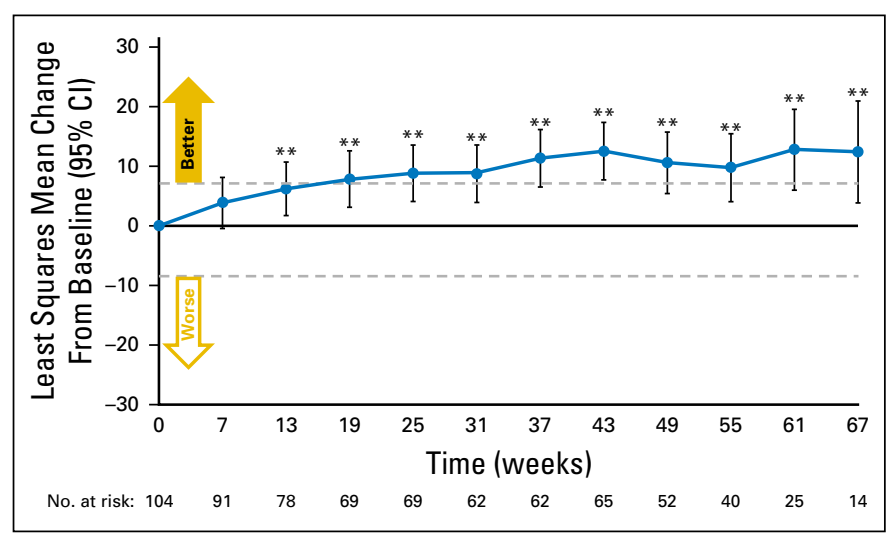

Fig A5. Patient-reported outcomes: mean change from baseline per three-level five-dimensional EuroQol instrument visual analog scale. $\left.{ }^{* *}\right) P<.01$.

\begin{tabular}{|lcr|}
\hline \multicolumn{3}{|c|}{ Table A1. ORR, Best Overall Response, and DCR per BICR (N = 119) } \\
\hline \multicolumn{1}{|c|}{ Response } & No. (\%) & $95 \% \mathrm{Cl}$ \\
\hline ORR & $58(49)$ & 39.5 to 58.1 \\
Best overall response & $5(4)$ \\
Complete response & $53(45)$ \\
Partial response & $39(33)$ \\
Stable disease & $17(14)$ \\
Progressive disease & $4(3)$ \\
Not determined & $1(1)$ \\
Not reported & $94(79)$ \\
Disease control for $\geq 12$ weeks & \\
\hline Abbreviations: BICR, blinded independent central review; DCR, disease control \\
rate; ORR, objective response rate.
\end{tabular}

\begin{tabular}{|c|c|c|}
\hline \multirow[b]{3}{*}{ Biomarker } & $\begin{array}{l}\text { efined } \mathrm{Pa} \\
\mathrm{nt}(\mathrm{N}=1\end{array}$ & opulations per \\
\hline & \multicolumn{2}{|c|}{ No. (\%) } \\
\hline & ORR & $\begin{array}{l}\text { Disease Control } \\
\text { for } \geq 12 \text { Weeks }\end{array}$ \\
\hline \multicolumn{3}{|l|}{ Tumor PD-L1 expression } \\
\hline$\geq 1 \%(n=26)$ & $14(54)$ & $20(77)$ \\
\hline$<1 \%(n=65)$ & $34(52)$ & $51(78)$ \\
\hline Unknown $(n=28)$ & $17(61)$ & $24(86)$ \\
\hline \multicolumn{3}{|l|}{ Mutation status } \\
\hline BRAFIKRAS wild type $(\mathrm{n}=31)$ & $17(55)$ & $24(77)$ \\
\hline$B R A F$ mutant $(\mathrm{n}=29)$ & $16(55)$ & $23(79)$ \\
\hline KRAS mutant $(\mathrm{n}=44)$ & $25(57)$ & 37 (84) \\
\hline Unknown $(n=15)$ & $7(47)$ & $11(73)$ \\
\hline \multicolumn{3}{|l|}{ Clinical history of Lynch syndrome* } \\
\hline Yes $(n=35)$ & $25(71)$ & $30(86)$ \\
\hline No $(n=31)$ & $15(48)$ & $25(81)$ \\
\hline Unknown ( $n=53)$ & $25(47)$ & $40(75)$ \\
\hline \multicolumn{3}{|c|}{$\begin{array}{l}\text { Abbreviations: DCR, disease control rate; ORR, objective response rate; PD-L1, } \\
\text { programmed death-ligand } 1 . \\
{ }^{*} \text { Lynch syndrome designation was based on the clinical records of the patients } \\
\text { at sites in countries where this reporting was permitted (excluded Italy). }\end{array}$} \\
\hline
\end{tabular}




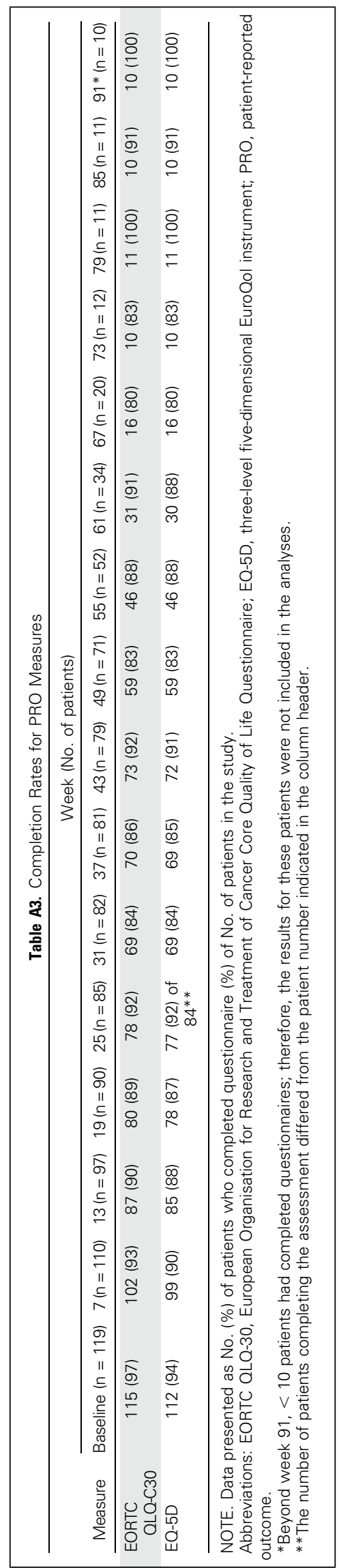




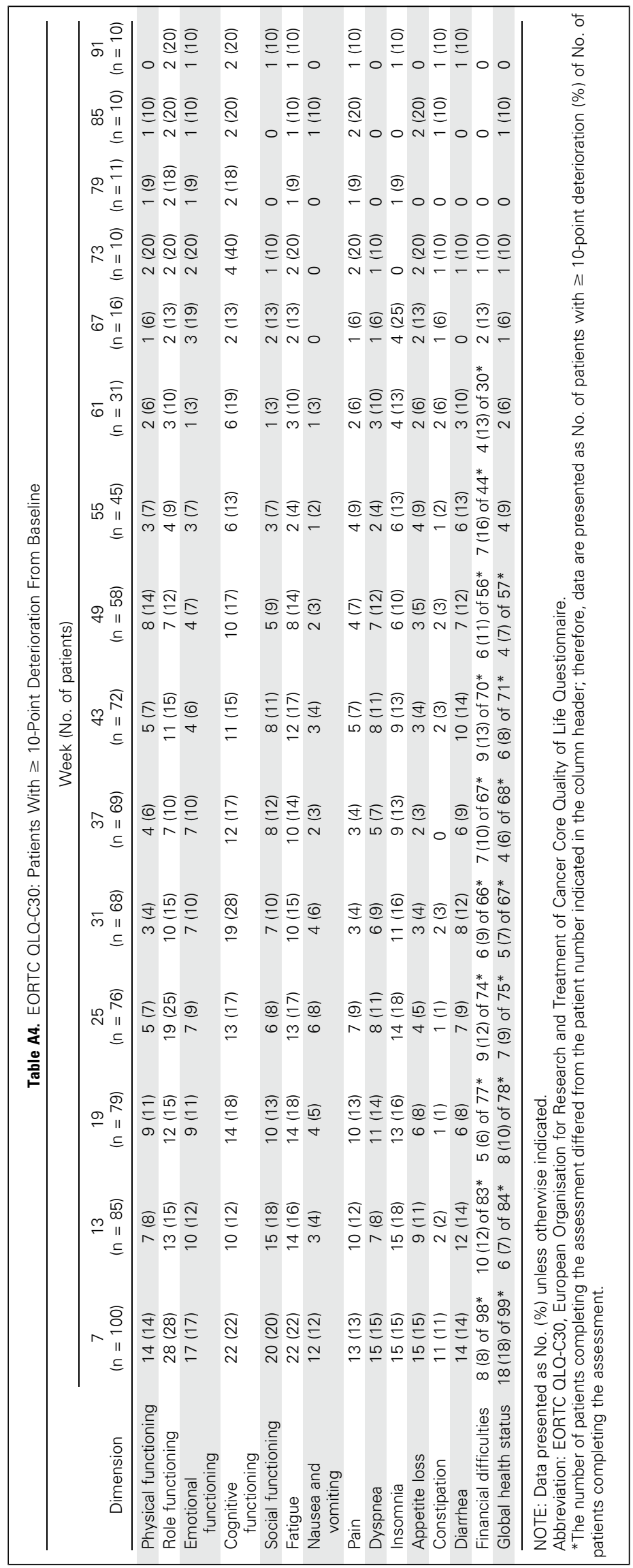




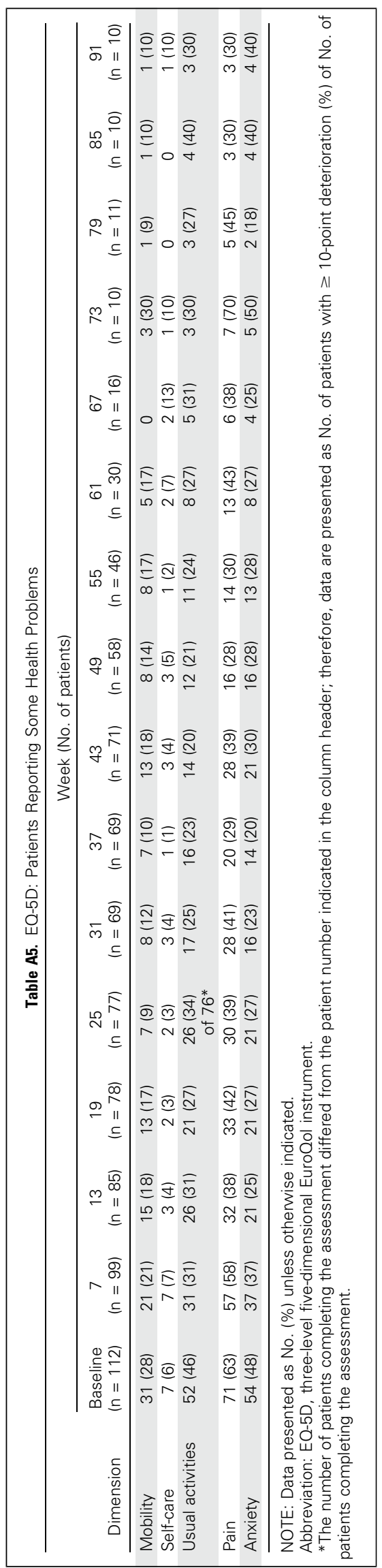


Overman et al

Table A6. Summary of Select Treatment-Related Adverse Events

\begin{tabular}{|c|c|c|c|c|c|c|c|c|c|c|}
\hline \multirow[b]{2}{*}{ Organ System } & \multicolumn{2}{|c|}{$\begin{array}{c}\text { Patients With Event } \\
\text { No. (\%) } \\
\end{array}$} & \multicolumn{2}{|c|}{$\begin{array}{c}\text { Time to Onset (weeks) } \\
\text { Median (range) }\end{array}$} & \multicolumn{2}{|c|}{$\begin{array}{l}\text { Patients With } \\
\text { Resolution } \\
\text { No. (\%) }\end{array}$} & \multicolumn{2}{|c|}{$\begin{array}{l}\text { Time to Resolution } \\
\text { Median (range) }\end{array}$} & \multicolumn{2}{|c|}{$\begin{array}{c}\text { Patients Who } \\
\text { Received Immune- } \\
\text { Modulating } \\
\text { Medication } \\
\text { No. (\%) } \\
\end{array}$} \\
\hline & Any Grade & $\begin{array}{l}\text { Grade } \\
3-4\end{array}$ & Any Grade & $\begin{array}{c}\text { Grade } \\
3-4\end{array}$ & Any Grade & $\begin{array}{c}\text { Grade } \\
3-4\end{array}$ & Any Grade & $\begin{array}{c}\text { Grade } \\
3-4\end{array}$ & Any Grade & $\begin{array}{c}\text { Grade } \\
3-4\end{array}$ \\
\hline Skin & 34 (29) & $5(4)$ & $5.2(0.3-38)$ & $2.1(1-23)$ & $24(71)$ & $5(100)$ & $9.0(0.4-106)$ & $2.6(1-13)$ & $19(56)$ & $4(80)$ \\
\hline Endocrine & $30(25)$ & $6(5)$ & $9.1(3-42)$ & $13.1(8-20)$ & $12(40)$ & $3(50)$ & Not reached (2-93) & Not reached (2-48) & $11(37)$ & $4(67)$ \\
\hline $\mathrm{GI}$ & $27(23)$ & $4(3)$ & $9.1(0.3-41)$ & $16.1(4-38)$ & $25(96)$ & $4(100)$ & $1.5(0.1-28)$ & $1.1(1-3)$ & $6(22)$ & $3(75)$ \\
\hline Hepatic & 23 (19) & $13(11)$ & $7.0(1-42)$ & $10.0(3-42)$ & $17(74)$ & $11(85)$ & $5.0(0.3-66)$ & $3.3(1-57)$ & $10(43)$ & $9(69)$ \\
\hline Pulmonary & $6(5)$ & $1(1)$ & $10.5(4-15)$ & $6.0(6-6)$ & $5(83)$ & $1(100)$ & $4.5(1-49)$ & $1.0(1-1)$ & $2(33)$ & 0 \\
\hline Renal & $6(5)$ & $2(2)$ & $12.6(1-36)$ & $15.6(7-24)$ & $5(83)$ & $1(50)$ & $6.3(3-21)$ & Not reached (4-21) & $2(33)$ & 2 (100) \\
\hline
\end{tabular}

